Document downloaded from:

http://hdl.handle.net/10251/117573

This paper must be cited as:

Marzal-Romeu, S.; Salas-Puente, RA.; González-Medina, R.; Garcerá, G.; Figueres Amorós, E. (2018). Efficient Event Notification Middleware for Smart Microgrids over P2P Networks. IEEE Transactions on Smart Grid. https://doi.org/10.1109/TSG.2018.2865432

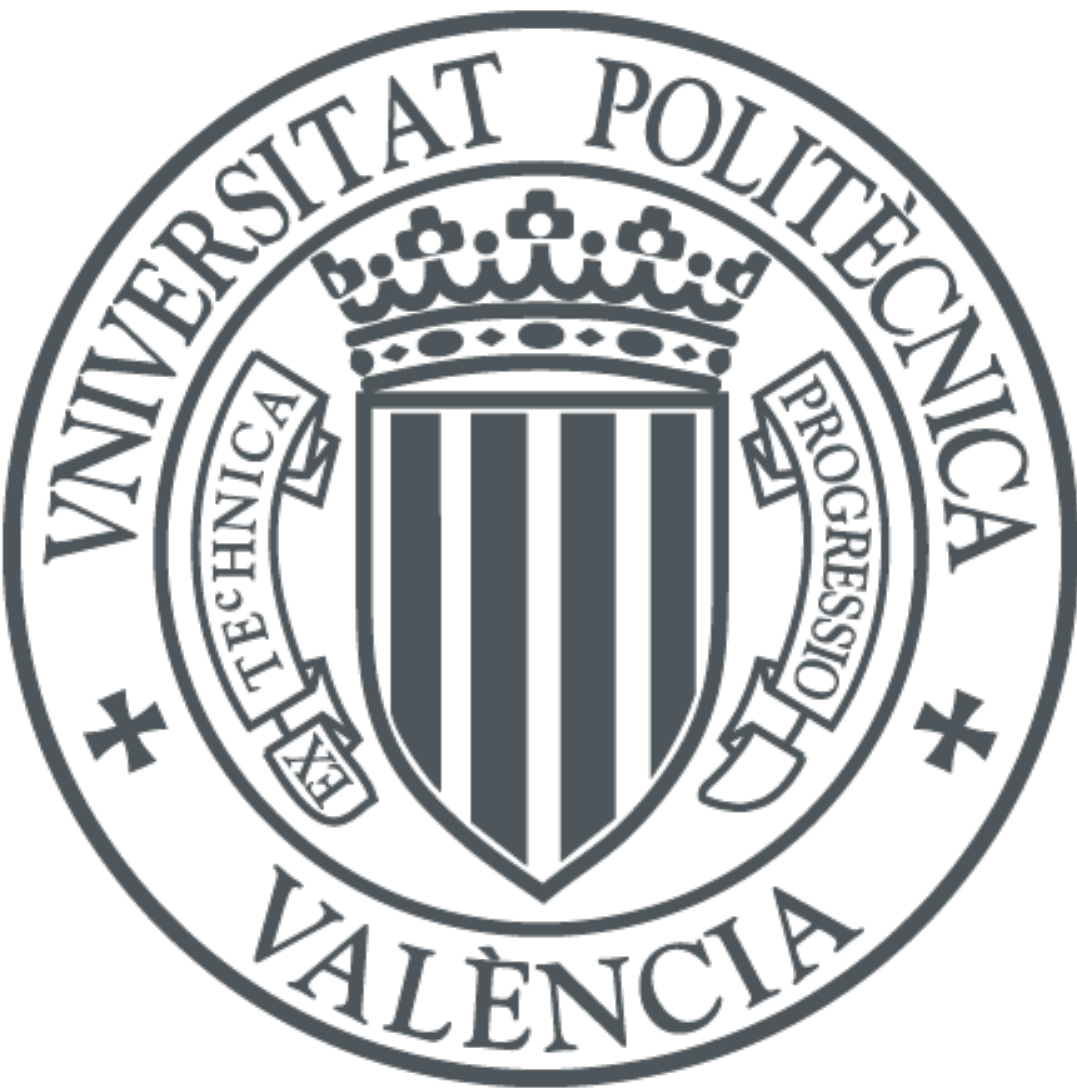

The final publication is available at

http://doi.org/10.1109/TSG.2018.2865432

Copyright Institute of Electrical and Electronics Engineers

Additional Information

(C) 2018 IEEE. Personal use of this material is permitted. Permissíon from IEEE must be obtained for all other uses, in any current or future media, including reprinting/republishing this material for advertisíng or promotional purposes, creating new collective works, for resale or redistribution to servers or lists, or reuse of any copyrighted component of this work in other works. 


\title{
Efficient Event Notification Middleware for Smart Microgrids over P2P Networks
}

\author{
S. Marzal, R. Salas-Puente, R. González-Medina, G. Garcerá, Senior Member IEEE, E. Figueres, \\ Senior Member, IEEE.
}

\begin{abstract}
Microgrids are moving towards large-scale smart distributed networks which demand an efficient and reliable communication infrastructure to manage, control and monitor energy resources. With regard to this, publisher/subscriber event-based middleware has become relevant for large-scale distributed time applications because it allows decouple time and space between senders and receivers. Particularly the content publish/subscribe systems over structured peer-to-peer (P2P) networks has emerged to enhance scalability and dynamism of notification middleware systems. However, this type of systems use multicast routing schemes that still generate much network traffic and as a consequence an overload of the communication channel is produced. This results in inefficient network utilization and rapid depletion of network resources leading to unreliable operations, degradation of system performance and even instability of the microgrid. In this paper, a new contentbased publish/subscribe notification middleware over structured P2P systems is proposed, such that smart microgrid communication requirements are met. This proposed system organizes the publications and subscriptions in a one dimensional representation using the Hilbert space filling curve. Through this representation, an innovative routing and matching algorithms are developed. Experimental results demonstrate that the proposed publisher/subscribe system significantly enhance efficiency of the system, network performance and the use of computational resources.
\end{abstract}

Index Terms-Notification middleware, P2P systems, microgrids.

\section{INTRODUCTION}

$\mathrm{M}$ icrogrids (MGs) are defined as groups of distributed energy resources (DERs) formed by distributed generation (DG), both renewable and/or conventional, energy storage system (ESSs) and loads. Microgrids can operate connected to the main grid via the point of common coupling (PCC) (grid connected mode) or completely separated from the main grid (island mode) [1]. The data transmissions among microgrid components are implemented via communication networks. Thus, the management and control

Manuscript received Month $\mathrm{xx}, 2 \mathrm{xxx}$; revised Month $\mathrm{xx}$, xxxx; accepted Month x, xxxx. This work is supported by the Spanish Ministry of Economy and Competitiveness (MINECO) and the European Regional Development Fund (ERDF) under Grant ENE2015- 64087- C2- 2 R. This work is supported by the Spanish Ministry of Economy and Competitiveness (MINECO) under BES- 2013- 064539.

S.Marzal, R. González-Medina, R.Salas-Puente, G. Garcerá and E. Figueres are with Grupo de Sistemas Electrónicos Industriales del Departamento de Ingeniería Electrónica, Universitat Politècnica de València, Camino de Vera s/n, 46022 Valencia, España; raugonme@upv.es; rosapue1@posgrado.upv.es;ggarcera@eln.upv.es, efiguere@eln.upv.es.

*Correspondence: silmarro@upv.es; Tel.: +34-96-3877007 (ext. 76021) of the microgrid by the Microgrid Control System, MCS, rely on a robust communication infrastructure [2]. One of the key points for integrating DERs into microgrids is the design of a control architecture that coordinates each one of the DER units. Traditionally, this control architecture is categorized into three major groups: centralized, decentralized and distributed control methods [3]. In this study a distributed

architecture is considered (Figure 1), in which the local nodes have its own MCS and are able to take decisions. In addition, nodes can share their information through two-way communication links [4].

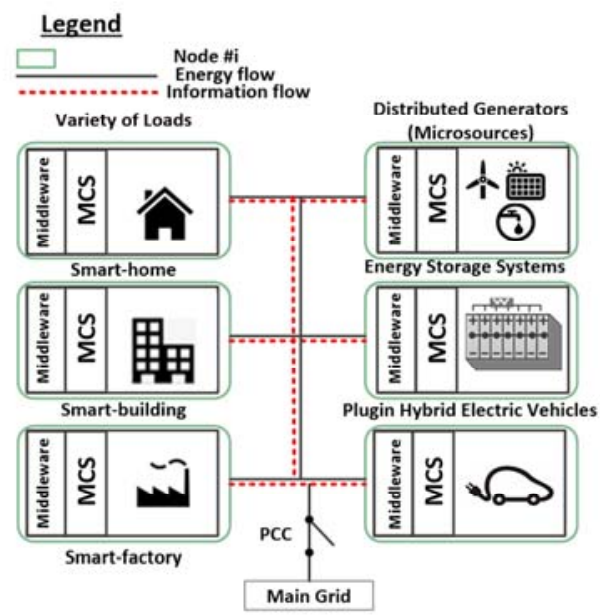

Fig.1. Overview of the distributed microgrid scenario

Nowadays, smart microgrids communications have to deal with large-scale distributed energy resources and manage them in real-time and dynamically. To cope with this, middleware architectures are one of the technologies adopted and they are a critical element in adapting to the challenges of smart microgrids [5]. In particular, microgrids require agile, flexible, real-time communication solutions, which have driven the growth of event-based middleware architectures. In an event-driven communication scheme, the signal transmission is triggered only in response to an event when a significant change of controlled or monitored physical variables occurs. A convenient way to construct microgrid services that use event infrastructures is through the implementation of communication middleware that works under a publish/subscribe (pub/sub) paradigm [6]. Pub/sub paradigm allows non-blocking asynchronous communications and one-to-many messages distribution for event notification.

In this regard, structured $\mathrm{P} 2 \mathrm{P}$ networks have become 
popular as a platform to develop event-driven pub/sub middleware for large-scale distributed systems. These networks have many advantages such as decentralization (central points are not needed), self-organization (nodes can dynamically arrive or depart) and scalability (available resources grow with the number of nodes in the network) [7],[8]. In fact, recent researches [9], [10] suggest the extension of the IEC 61850 communication standard for microgrids to include $\mathrm{P} 2 \mathrm{P}$ communication systems aiming to better adapt to the new large-scale distributed scenario of microgrids.

Existing pub/sub systems based on structured P2P networks, traditionally use topic-based systems for publishing event notifications and multicast protocols for routing this event notification [11]. However, the use of these techniques is not optimal for microgrids because its network requirements are more demanding [12]. The reasons for that are the following: on one hand, in topic-based pub/sub systems each event is labeled with a topic (predefined subjects). The main disadvantages of the topic-based model are the limited flexibility and accuracy it offers to subscribers. As a result, a subscriber has to receive all the notifications relative to a topic though the subscriber might be interested in only a subset of the events. In addition, topic-based systems give limited choices of subscriptions while in microgrids applications, the subscribers must be able to specify their interests more accurately using a set of predicates, leading to a more optimal and efficient control [13]. On the other hand, multicast routing generates a large network traffic which can produce congestion, increased delays and poor bandwidth utilization. Moreover, with multicasting a high amount of false positives could take place, due to an event could be transmitted to nodes that are not interested in it or not need to route it to other destinations [11]. The pub/sub systems that work in environments where vital information is transmitted, false positives are not acceptable because the efficiency of the whole system could be significantly reduced [14].

Furthermore, communications between DERs is carried out through IEDs (Intelligent Electronic Devices). IEDs are devices equipped with operating systems that make two-way communication possible to monitor and manage the power grid. However, the main problem to their use is the limited computational capabilities. Given the event-driven middleware should be embed in each node of microgrid, the computational resources used for the middleware operation should be minimized [15].

Summarizing, conventional pub/sub notification-based middlewares are not properly adapted for microgrid environments due to the following reasons: they not offer subscription flexibility, they produce a high amount of network traffic as well as high network latencies; finally, they need high rates of computational resources. Therefore, the development of an event notification middleware specifically designed for microgrids is needed, which should be adapted to the quality performance requirements of this kind of applications.

In this paper a new and efficient event notification middleware architecture based on pub/sub content-based system over structured $\mathrm{P} 2 \mathrm{P}$ networks particularly built for smart microgrids is proposed. The main contributions of this work are: (i) a Hilbert mapping system which allows subscribers defining multiple dimensions of the contents in a single dimensional space by using a set of ranges (this allows for greater subscriptions flexibility and robustness in contrast with topic-based systems that are restricted to predefined topics), ii) an efficient content matching algorithm to reduce false positives rates and speed-up routing decisions and iii) a routing protocol based on ranges representation, ranged based routing protocol (RBR) instead of multicast routing. The proposed protocol reduces the network traffic, as the pub/sub system can transmit an event only to the subscribers that are concerned in.

These contributions allow a greater network performance and also save computational resources. The reduction of the network traffic involves a low number of messages transmitted so the CPU utilization decreases while the subscriptions optimization reduces the communication overhead, which reduces the memory usage.

The paper is organized as follows. In Section 2, the challenges of event notification middleware for MGs are provided. In section 3, the proposed solution is described. In Section 4, experimental results are presented and analyzed. Finally, Section 5 provides some conclusions.

\section{Challenges of EVEnt NOtificAtion-BASED MIDDLEWARE FOR MICROGRIDS}

To avoid unnecessary information request, an event notification service (ENS) that use event-based communication model can be used in microgrids. This service will be able to monitor and control the power network in an efficient manner as this communication architecture reduces the communication and computation load [16],[17]. In this technique, the pub/sub communication paradigm is mainly used to notify system changes to a set of interested receivers. In a pub/sub system, the providers of information (publishers) disseminate events to the system and the information consumers (subscribers) subscribe to the event that are interested on. All data are published or subscribed to/from the middleware service domain (Figure 2). When the middleware receives a publication it searches for matching subscriptions and notify them, and then the required data communication is achieved [18]. Relative to subscription models, the data distributed through the middleware service can be topic-based or content-based. On the one hand, in topic-based systems, the subscribers define its interest for a specific topic and will receive messages regarding to that topic. For instance, in the case of microgrids topics could be critical loads, photovoltaic generators, storage devices, island mode, etc. On the other hand, in content-based system, subscribers can define conditions over the content of the topic. For example, under the topic "Photovoltaics generator" it may be defined a certain number of attributes. A set of three attributes of interest could be Power (supplied to the grid), DC voltage (at the PV field) and State (connected or disconnected). As it can be deduced, 
content-based pub/sub is a more robust architecture, as subscribers can select its filtering criteria through the definition of multiple dimensions of the message contents [19]. Note that topic-based systems are restricted to predefined subject fields.

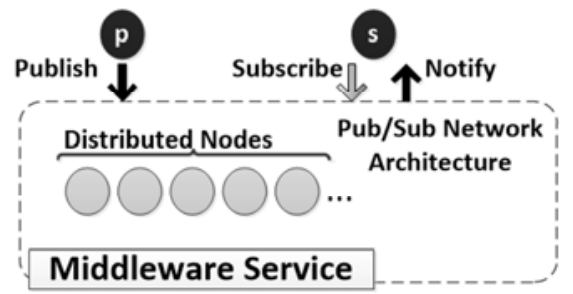

Fig.2. Communication architecture of a publish/subscribe system. (p and $\mathrm{s}$ indicate a generic publisher and subscriber respectively)

Event driven data exchange is an essential requirement for future self-manageable microgrid implementations [20]. In fact, the IEC61850 standard promotes the development of pub/sub services. However, microgrids are growing and managing a great amount of DERS. Therefore, some IEC 61850 components could be inadequate for Smart Microgrid (SMGs) development. In this sense, IEC 61850 standard should be extended to include large distributed control architectures implementing $\mathrm{P} 2 \mathrm{P}$ systems [9]. This goal is indispensable for a system that can be continuously changing, such as a smart microgrid. Regarding to that, a general overview of middleware platforms for smart grids is presented in [5], [15], [21], [22], [23], [24]. These works analyzes the key challenges associated with implementing services in the middleware layer. It is concluded that event notification middleware architectures for SMGs have various challenges:

1. Real-time distributed pub/sub protocols which enable P2P communications [25][26]: P2P communication architecture provides a more reliability and suitable environment for distributed devices as it permits selfdiscovery of new nodes/devices and self-organization. Due to this, the dynamic participation of the nodes/devices in the network is allowed and it is especially important for microgrids, in which a large number of devices (electric vehicles, loads, etc.) can be continuously joining and leaving the microgrid.

2. Flexible subscription system: Moving the subscription model from topic-based to content-based improve the system flexibility and accuracy [18],[27].

3. Reduced computational resources consumption: To make feasible the Smart Microgrid, power devices deployed in it are equipped with IEDs. They are usually embedded systems with limited computing resources. The limited nature of the embedded system resources, especially memory size and CPU, complicates meeting the real-time constraints [28]. Since event-driven middleware must be embedded in each node of the microgrid, the computational resources used for the middleware operation should be minimized.

4. Network quality requirements: To guarantee the network stability face to certain events, the microgrid control system should monitor all the network elements and act accordingly when a parameter exceeds the specified thresholds. To achieve that, data must be delivered promptly. In microgrids, latency and bandwidth are essential to meet the microgrid requirements [29] following IEC 61850 and IEEE 1646 standards (See Table I) [30], [31].

Based on the described above, some solutions have been proposed in [32], [33] to address some of these challenges. In [32] a pub/sub middleware for microgrids is developed. Similarly, in [33] the authors present a novel middleware framework (GridStat) for the power grid operation. As performance indicators, they use the latency and load scalability parameters. Both studies propose a pub/sub communication architecture for event notification middleware. However, they use agents instead of P2P nodes. Although agents can be structured forming decentralized topologies, they cannot act simultaneously as servers and clients, which results in a lack of agent proactivity. The connectivity between them is not carried out by creating virtual links (overlays), which cause lack of self-discovery and self-healing capabilities [34]. To overcome these limitations, [25], [26] propose a Data Distribution Service (DDS) middleware. This kind of middleware uses Pub/Sub communications over P2P networks. Furthermore, [26] performs a comparative analysis of the latencies and bandwidth measurements of the DDS middleware. However, in both studies, the pub/sub system is topic-based and the routing of events is carried out by multicast. In general, the existing P2P pub/sub systems are usually created by using any kind of structured overlay networks. These structured systems are called DHT's (Distributed Hash Table) because their use tables for routing. Examples of DHT networks include Chord, CAN, Tapestry, etc. Using a DHT-P2P solution, the information can be sent by multicasting. The use of a multicast routing protocol for the pub/sub system has been the natural choice in topic-based DHT systems, as each published topic is identical to one multicast group. However, multicasting cannot be directly used in content-based systems because subscribers cannot be directly mapped to multicast groups [35], [36]. Moreover, when a multicast routing is used the data is replicated and sent to all the nodes in the list. The multicast process and data replication introduce high latencies and also high traffic that can be not suitable for microgrids.

TABLE I

NETWORK REQUIREMENTS FOR MGS APPLICATIONS

\begin{tabular}{|c|c|c|}
\hline $\begin{array}{l}\text { Communication } \\
\text { Requirement }\end{array}$ & Latency & Bandwidth \\
\hline $\begin{array}{c}\text { Distribution } \\
\text { Management }\end{array}$ & $100 \mathrm{~ms}-2 \mathrm{~s}$ & $9.6-100 \mathrm{kbps}$ \\
\hline Demand Response & 500ms-several minutes & $\begin{array}{c}\text { 14-100kbps per } \\
\text { node }\end{array}$ \\
\hline Monitoring Information & $15 \mathrm{~ms}-200 \mathrm{~ms}$ & $9.6-56 \mathrm{kbps}$ \\
\hline Control Information & $16 \mathrm{~ms}-100 \mathrm{~ms}$ & $9.6-56 \mathrm{kbps}$ \\
\hline $\begin{array}{l}\text { Messages requiring } \\
\text { immediate actions }\end{array}$ & $\begin{array}{l}\text { 1A: } 3 \mathrm{~ms} \text { or } 10 \mathrm{~ms} ; 1 \mathrm{~B}: \\
20 \mathrm{~ms} \text { or } 100 \mathrm{~ms}\end{array}$ & $9.6 \mathrm{kbps}$ \\
\hline Outage Management & $2 \mathrm{~s}$ & $56 \mathrm{kbps}$ \\
\hline
\end{tabular}

The multicast routing problem has been studied in some research works [11],[17],[27] looking for clusters of nodes configuration for multicast groups in order to reduce the network traffic. In these solutions, the routing system allows 
each node to create a routing table that associates the subscription of interest. Thus, each node is incorporated to the clusters that contain its subscription information and each publisher transmits a notification through the group of clusters. Upon publishing an event, the node transmits a notification to the nodes that are in its routing table and simultaneously are interested in that topic, as well as those nodes that operate as a relay node for the topic. The matching system of these solutions works as follows: once the node receives the notification, it extracts the information, filters the content and subscribes if the content matches.

However, refs [11] and [27] are topic-based and they are solutions that have not been specifically developed for energy environments. Therefore, the network performance and the optimization of computational resources have not been taken into account. In addition, although [17] is developed for energy environments, the pub/sub system is also topic-based and is not as efficient as content-based, due to there is not guarantees that subscribers receive accurate information about the topics they are interested in. As a consequence, the routing system still generates an excessive traffic as an event can be sent to not interested nodes and they do not need to route it [9]. In addition, the topic-based system also influences in the matching system that could produce too much false positives.

To overcome this limitation, in [14] an indexing system to provide content-based pub/sub systems over P2P networks is presented. A one dimensional representation using Hilbert space filling curve for pub/sub system is created. The results indicate that with Hilbert indexation, subscription forwarding load and speed up content matching is reduced. However, this system is neither a solution for microgrids nor routing mechanisms are proposed. In fact, this solution has been developed for file sharing applications in P2P environments. However, in such applications, the network performance requirements are less critical than the ones of microgrids. For that, this system is not a suitable solution for microgrids.

In summary, the previously proposed solutions that develop pub/sub middlewares for energy networks have some common limitations: (i) their focus is on the theoretical background of middleware architectures without any performance analysis; (ii) their studies are constrained to topic-based systems which use multicast routing mechanisms, with no attention to the network performance cost or the wasted computational resources; and (iii) neither the content pub/sub system over P2P networks nor their routing mechanism have been evaluated for MGs. Table II summarizes these conclusions. Therefore, the best pub/sub communication system to develop an optimal event notification middleware for microgrids seems to be a P2P content-based pub/sub system. For this reason, this paper is focused on developing such kind of middleware architecture for microgrids.
TABLE II

REVIEW ON PUB/SUB MIDDLEWARE ARCHITECTURES FOR ENERGY NETWORK

\begin{tabular}{|c|c|c|c|c|c|c|}
\hline$\dot{\ddot{\mathscr{u}}}$ & 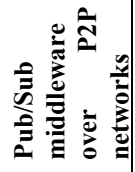 & 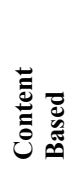 & 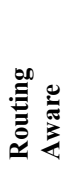 & 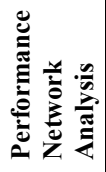 & 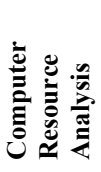 & 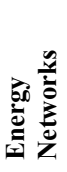 \\
\hline$[32],[33]$ & - & - & - & $\bullet$ & - & $\bullet$ \\
\hline$[25],[26]$ & $\bullet$ & - & $\bullet$ & $\bullet$ & - & $\bullet$ \\
\hline$[11],[27]$ & $\bullet$ & - & $\bullet$ & - & - & - \\
\hline [17] & $\bullet$ & - & $\bullet$ & $\bullet$ & - & $\bullet$ \\
\hline [14] & $\bullet$ & $\bullet$ & - & $\bullet$ & - & - \\
\hline $\begin{array}{l}\text { This } \\
\text { study }\end{array}$ & • & $\bullet$ & $\bullet$ & $\bullet$ & $\bullet$ & $\bullet$ \\
\hline
\end{tabular}
specified in the column heading. - Indicates that it does not

\section{Proposed Event Notification Middleware}

The proposed system is a content-based pub/sub event notification middleware over a P2P Chord overlay protocol. It is composed by the following components:

(A) An indexing scheme to implement a content-based pub/sub over P2P networks to improve the system flexibility and accuracy.

(B)A content matching subscription mechanism for reducing the number of false positives.

(C)An optimized routing engine (RPL protocol) for event dissemination system, to accomplish microgrid network performance requirements.

(D)An event notification service application for achieving a fully distributed system.

Figure 3 presents the architecture of the proposed system.

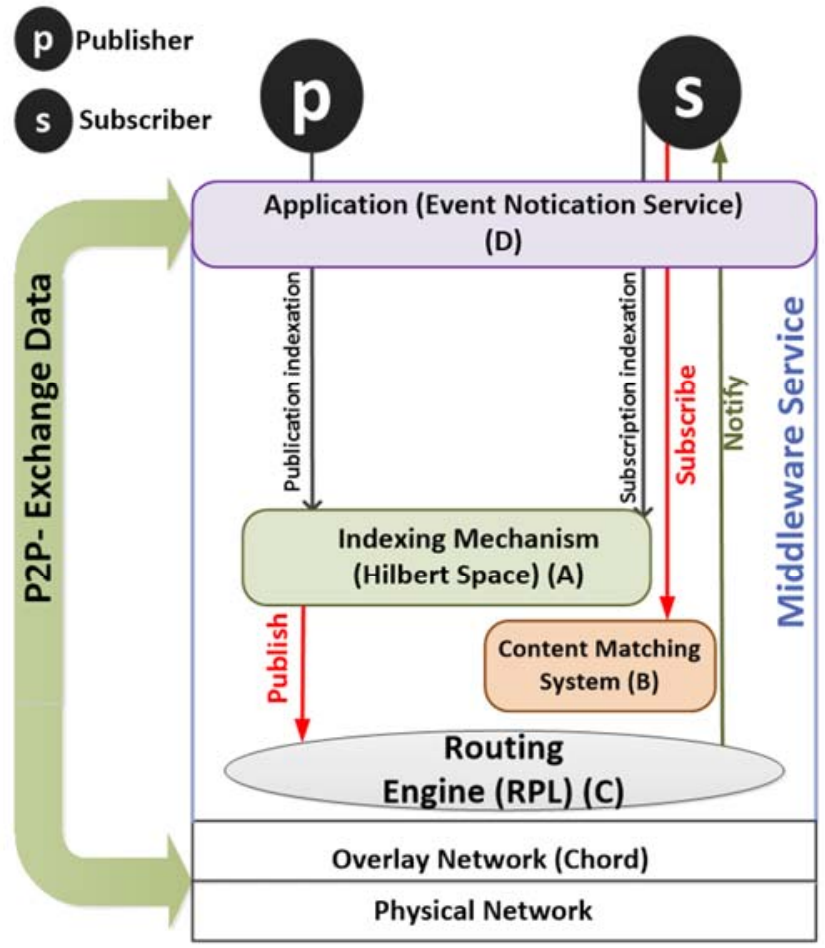

Fig.3. Overview of the proposed architecture 


\section{A. The indexing mechanism construction}

For content-based communication in a pub/sub system, a crucial component is the index space, as it is responsible for mapping the data elements to this index space. To be able to effectively support the subscription management, the multidimensional content space has been associated to a single dimensional space by means of the Hilbert Space Filling Curve (Hilbert-SFC) [37].The $d-$ dimensional space can be seen as a d-dimensional cube and the SFC is recursively constructed. For that, the $\mathrm{d}-$ dimensional cube is first partitioned into $2^{\mathrm{k}}$ intervals and these intervals are numbered from 0 to $2^{\mathrm{k}}-1$. As a result of the cube partitioning, $\left(2^{\mathrm{k}}\right)^{\mathrm{d}}$ cubes of $\mathrm{d}-$ dimensional are obtained. Each one of the cubes is called cell [14]. The partitioned ddimensional space is represented by $\mathrm{H}_{\mathrm{k}}^{\mathrm{d}}$. The index of each cell has been produced by using the Hilbert space filling curve and it is represented as $\mathrm{C}=\left(\mathrm{x}_{1}, \mathrm{x}_{2}, \ldots . \mathrm{x}_{\mathrm{d}}\right)$ where $\mathrm{x}_{\mathrm{i}}$ is an integer of the Hilbert key in the partitioning space [38]. To illustrate this, the drawings of the first, second and third order approximation of the Hilbert curve have been depicted in Figure 4, corresponding to: $\mathrm{H}_{1}^{2}, \mathrm{H}_{2}^{2}, \mathrm{H}_{3}^{2}$, respectively. In Figure 4 (a) and 4 (b), the Hilbert cells have been indexed in binary, while in Figure 4 (c) they have been indexed in its corresponding integer Hilbert key in the partitioning space.

By applying the Hilbert mapping to this multidimensional space, the proposed system maps each one of the ddimensional attributes to a point of the SFC. This feature achieves high expressiveness in subscriptions as well as the reduction of the average message size for event publication and subscriptions. In this way, a reduction of both communication overload and latencies is achieved. The publication and subscription indexing are detailed below.

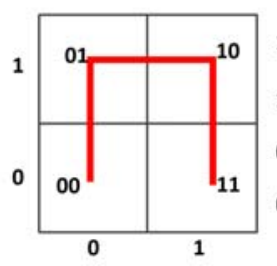

(a)

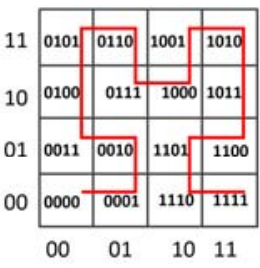

(b)

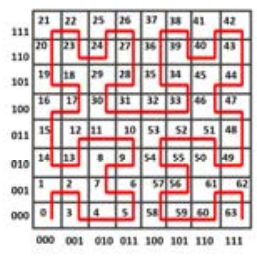

(c)
Fig.4. (a) $\mathrm{H}_{1}^{2}$, (b) $\mathrm{H}_{2}^{2}$, (c) $\mathrm{H}_{3}^{2}$. The assignment of keys to cells in a Hilbert Space: first, second and third order.

\section{1) Events Publication}

In the proposed solution, every event publication ' $p$ ' belongs to the event space $H$ and has an event type ' $\tau$ '. It is defined as follows:

$$
(p: \tau) \in H
$$

An event type $\tau$ has the following definition; $\tau=$ $\left(\mathrm{n}_{\tau},\left\{\mathrm{a}_{0}, \mathrm{a}_{1}, \ldots \mathrm{a}_{\mathrm{d}-1}\right\}\right)$ where $\mathrm{n}_{\tau}$ represents the event type name and $\left\{a_{0}, a_{1}, \ldots a_{d-1}\right\}$ is a collection of event attribute types. Each attribute has its own value, $v_{i}$, in such way that every publication (event), p, symbolize a point in the content space and can be defined as:

$$
p: \tau=\left\{\left(a_{0}, v_{0}\right),\left(a_{1}, v_{1}\right), \ldots\left(a_{d-1}, v_{d-1}\right)\right\}
$$

\section{2) Events Subscription}

A variant of content-based publish/subscribe is proposed. Through this modification, the subscribers specify their interest in two steps. Firstly, the event type is designated and secondly a collection of predicates regarding this event are defined. Thus, an event subscription ' $s$ ' has an event type ' $\tau$ ', which is a collection of predicates with its attributes.

$$
\mathrm{s}: \tau=\left\{\mathrm{r}_{1}, \mathrm{r}_{2}, \ldots, \mathrm{r}_{\mathrm{k}}\right\}
$$

The set $\left\{r_{1}, r_{2}, \ldots, r_{k}\right\}$ represents a conjunction of predicates. A predicate ' $r$ ' is a tuple, $r=\left(a_{r}, v_{r}\right)$ where $a_{r}$ is the attribute name and $v_{r}$ is the attribute value. Thus, each predicate represents the subset of the corresponding attribute domain in which the subscriber is interested in. The bounds of the subscription for $i_{\text {th }}$ attribute are defined by each predicate. Therefore, a subscription is represented by a rectangle that is composed by cells in the partitioned space.

Figure 5 illustrates an example of the proposed indexing pub/sub system in the two dimensional Hilbert space. Let us consider that three publications $\mathrm{p}_{1}, \mathrm{p}_{2}, p_{3}$ are generated and represented as 4,57 and 55 respectively. Let us also consider that a certain subscription ' $\mathrm{s}$ ' is given by the set of cells $\{4,5$, $6,7,56,57,58$, and 59\} (the blue rectangle in Figure 5). As it can be seen $\mathrm{p}_{1}$ and $\mathrm{p}_{2}$ are events of interest for such subscription while $\mathrm{p}_{3}$ will be ignored.

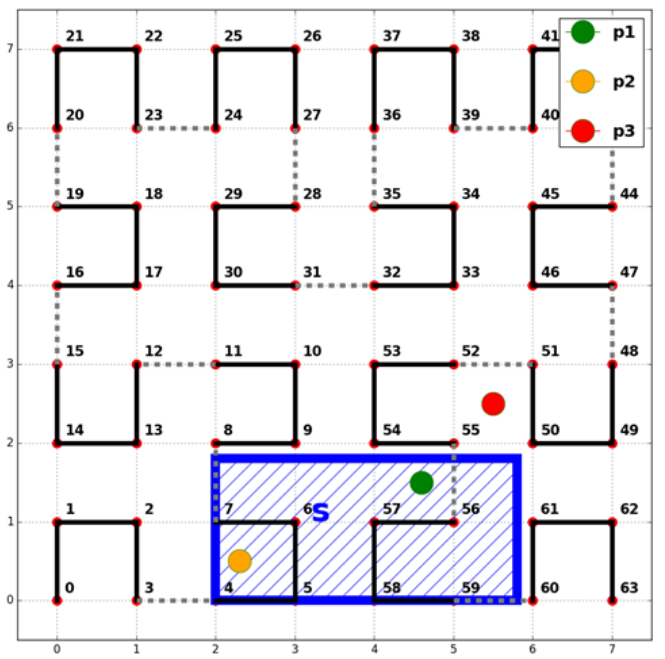

Fig.5. Example of publications and subscription indexing in a two dimensional space, $\mathrm{H}_{3}^{2}$

\section{B. Content Matching System}

A subscriber can have multiple subscriptions of interest. However, the management of large amount of information regarding subscriptions could lead to a big size of the subscriptions table. Big size of subscription tables results in a waste of processing resources and memory requirements as well as a large amount of redundant traffic. In order to avoid the unessential subscriptions dissemination and to reduce the size of the subscription tables, a subscription merging technique based on [14] has been implemented. Merging is a method that gives the minimum filter that represents a collection of subscriptions defined in the content space. Formally, a subscription $\mathrm{S}$ is a merger of set of subscriptions: 
$S_{1}, S_{2} \ldots S_{n}$ if $S \supseteq \bigcup_{i=1}^{n} S_{i}$.

Finding the minimum-bounding rectangle that comprises a set of subscriptions is the simplest way to achieve the subscription merging [14]. Since each subscription is represented by the boundaries of each attribute, a subscription can be represented as a d-dimensional rectangle in the content space. The result of merging is a new rectangle, $S_{-a u g}$, that includes all the original subscriptions, $\mathrm{S}_{\text {or }}$, that are being merged. Figure 6 shows the result of merging applied to three subscriptions in a $\mathrm{H}_{3}^{2}$ Hilbert space by means of minimum bounding rectangle. The merging rectangle is the minimumbounding rectangle $\left(\mathrm{S}_{\text {aug }}\right)$ that contain those cells that intersect with the original subscriptions and it is defined as follows:

$$
\begin{aligned}
& S_{-a u g}=\left\{\mathrm{C}\left(x_{1}, x_{2}, \ldots, x_{d}\right) \in H_{k}^{d} \text { where } C\left(x_{1}, x_{2}, \ldots, x_{d}\right) \cap\right. \\
& \mathrm{S} \neq \emptyset\}
\end{aligned}
$$

In this sample, the resulting augmented subscription $S_{\text {aug }}$ is the rectangle that has intersection with the original subscriptions. Therefore, the set off cells that form the augmented subscription, $S_{\text {aug }}$, is

$\{4,5,58,59,7,6,57,56,8,9,54,55,11,10,53,52\}$.

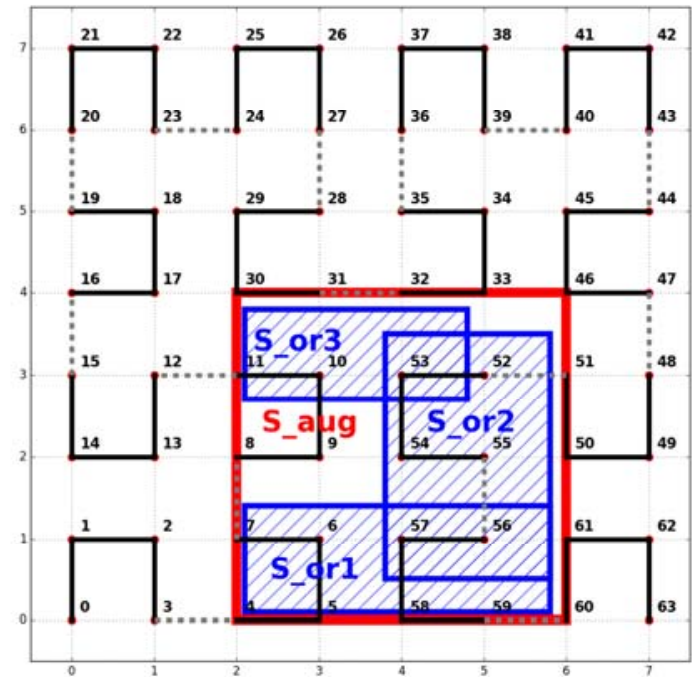

Fig.6. Merging of three original subscriptions $\mathrm{S}_{\text {_or }}$ in the augmented subscription $\mathrm{S}_{-a u g}$ indexed in the partitioned space, $\mathrm{H}_{3}^{2}$

Once the indexing cells of the augmented subscription are identified, the intervals that represent these cells must to be found. These set of intervals (ranges) are based on the Hilbert indexing space that compose the augmented subscription. As an example, the set of cells that form the augmented subscription considered in Figure 6 results in the following two intervals for representing the subscription: $\{[4,11],[52$, 59]\}. In this way, after receiving a publication, the system verifies the augmented subscription ranges. As it can be seen, the area of the augmentation subscription comprises some fragments of the content space that are not included in the original subscription. As a consequence, false positives may be generated when a publication is disseminated. This number of false positives is considerably small, due to the minimum surface area of the augmented subscription is taken.

\section{RBR Protocol (Ranged-Based Routing Protocol)}

As explained in section I, P2P generates high network traffic rates, so the conventional events dissemination-routing engines are not useful in the case of microgrids. For this reason, a Ranged-Based Routing (RBR) Protocol has been developed. The process of event routing is necessary for achieving an efficient event notification middleware. The main goal of the proposed routing engine is to meet the microgrid network performance requirements. For that, it is essential to find the cluster of nodes whose subscription matches with the given event. This avoids unnecessary retransmissions and reduces the network traffic.

The middleware has been developed over structured (DHT) P2P networks. The DHT protocol preferred for this work has been the Chord protocol, whose features are shown in Table III [39]. The basic idea of Chord is the following. The Chord DHT overlay arranges peers on a circle topology ranging from 0 to $2^{\mathrm{m}}-1$, where $\mathrm{m}$ is the number of bits of the identifiers. The peers are ordered following their identifiers. A key is stored in each node. The key identifier space assigns the keys between each one of the nodes and their predecessors. Each node in the circle also upholds a routing table, called finger table, which contains information about other neighbors (predecessor and successor) in the identifier space. The neighbor nodes that comprise the routing table have been selected deterministically, using an overlay-specific metric. The routing table is used by the lookup algorithm for efficient routing. Figure 7 shows an example of Chord overlay network.

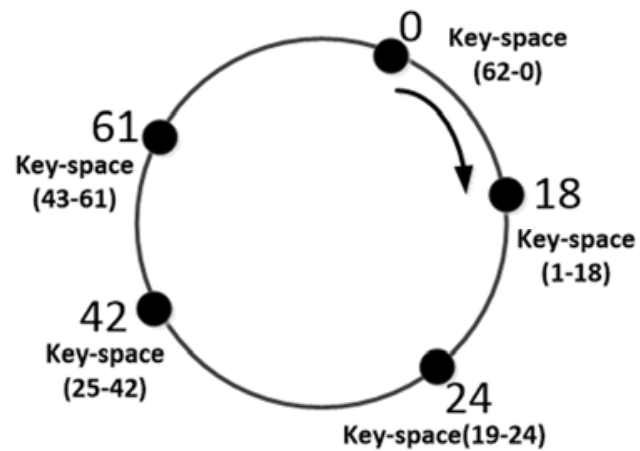

Fig.7. Example of DHT Chord overlay network with an identifier space $\left[0,2^{6}\right)$.

TABLE III

CHORD ROUTING PROTOCOL FEATURES

\begin{tabular}{|l|l|}
\hline \multicolumn{2}{|c|}{ Properties } \\
\hline Popularity & $\begin{array}{l}\text { Chord is the most popular DHT routing } \\
\text { protocol }\end{array}$ \\
\hline Design & $\begin{array}{l}\text { Reduce the complexity in designing P2P } \\
\text { systems. }\end{array}$ \\
\hline $\begin{array}{l}\text { Large- } \\
\text { distributed } \\
\text { system }\end{array}$ & $\begin{array}{l}\text { Allows developing completely distributed } \\
\text { systems. Nodes have the same responsibility in } \\
\text { the system }\end{array}$ \\
\hline Scalability & Is able to scale a large number of nodes \\
\hline $\begin{array}{l}\text { Self- } \\
\text { discovery }\end{array}$ & $\begin{array}{l}\text { Allows nodes be located even if the network is } \\
\text { highly dynamism and nodes enter and leave } \\
\text { continuously }\end{array}$ \\
\hline $\begin{array}{l}\text { Self- } \\
\text { organization }\end{array}$ & Nodes failures and disconnections are detected \\
\hline
\end{tabular}

Once the P2P layer is defined, the routing mechanism can be executed. On one hand, subscriptions nodes must to be 
initialized and the subscriptions must to be mapped to each Chord peer identifier. For that, the node's finger table of the traditional Chord algorithm has been modified in order to embed the augmented subscription ranges and keep the neighbor in the identifier space. Thus, in this scheme, when a subscriber node joins the network, it generates its identifier, type of events which it is interested in and the ranges of each event. After that, it sends the data to its neighbors. Hence, the subscriptions of the peers are mapped to each Chord peer identifier. On the other hand, when the publisher node is initialized, it creates two threads, one for ingoing and other for outgoing routing petitions, and remains waiting for an event. When an event is generated, the outgoing process is started and the event can be disseminated through the entire ring. The major challenge is how the event can be routed to the nodes whose content space overlaps the range of subscription. The proposed routing procedure developed for this system is shown in Figure 8.

This process is started when a publisher publishes an event in order to find subscriber nodes whose subscription matches with the given event. To achieve that, firstly, the event is indexed by using the Hilbert content space. Secondly, the event message must to be created. The event message has three main fields: i) The IP address of the node that sends the event, ii) the indexed event information, and iii) the list (Match_Node_List) with the matched subscribers nodes, which is generated as the event message progresses among the nodes of the ring. To build up this list, the Match_Search_Node process starts (Figure 9). For that, the node consults its finger table, which has been modified with regard to conventional Chord, to contain information about: i) the event type of each subscription $\left(\tau_{i}\right)$, ii) the ranges of this subscription $\left(R_{i}\right)$, and the link $\left(\mathrm{L}_{\mathrm{i}}\right)$ that contains the IP address and identifier (id) of the node $n_{i}$. Therefore the routing table has the entries $\left(\tau_{i}, R_{i}, \mathrm{~L}_{\mathrm{i}}\right)$. To determine the nodes subscription match, the node searches in its routing table and it verifies the type of event. If the event type matches to the one that the node is looking for, then it checks if it's subscripted to the subscription ranges of that node. If both of these conditions are met, the event message will be encoded and it will be sent to all matched nodes. Through sending the node match list, the receiver nodes is able to verify if the message has already been sent to the destination before to send it again, avoiding redundant searched messages.

Upon receiving an event, if the node is one of the subscribers, it decodes the message and then the matching process starts. In this process, the event is matched against all the subscriptions ranges stored in it and, if the event is not a false positive, it will subscribe to it. The searching process continues until the source's node is reached. On the contrary, if the event message reaches a publisher node (ingoing routing petition), before decoding the message, it verifies if is the source node. If not, it decodes the message and continues delivering it among the nodes of the ring until the node source is reached. The process finish when the source node is reached.

The scalability of the system is achieved using the node match list that is stored in the nodes, avoiding unnecessary traffic in the network. The objective is to identify the clusters; in this way, each node that contains matching subscribers receives one message per event, minimizing both communication and computational costs.

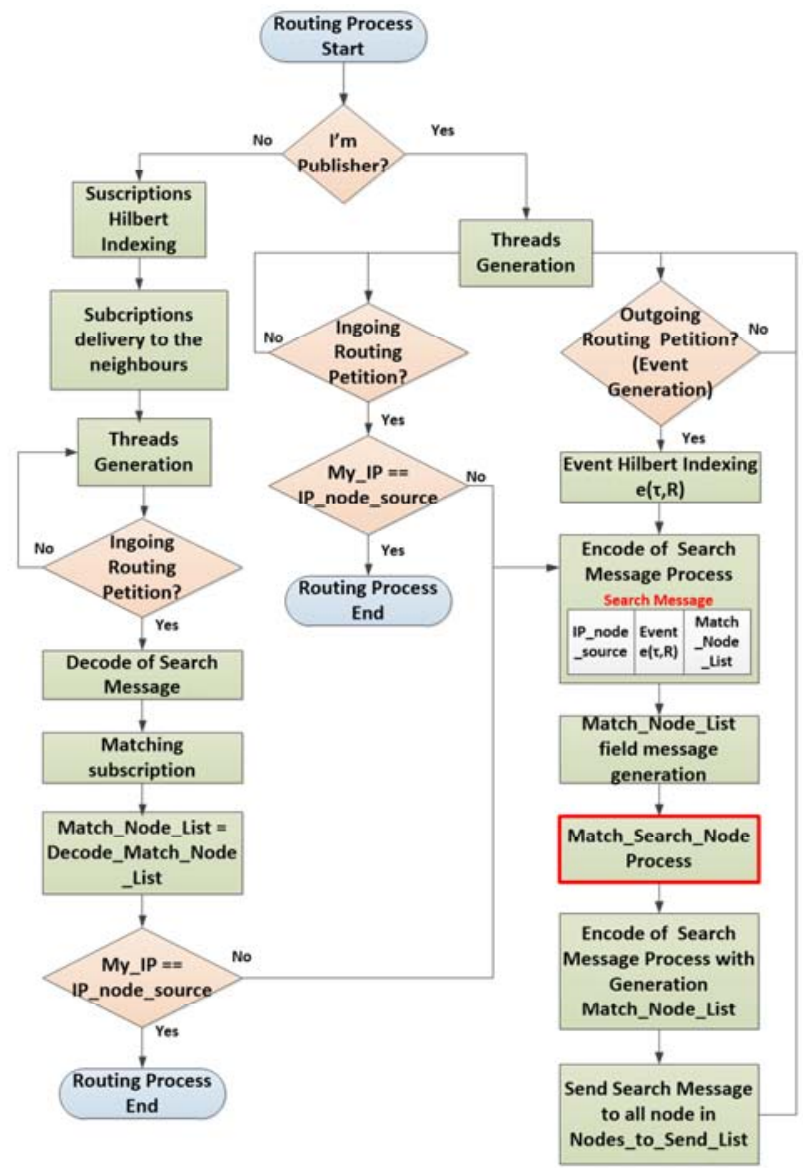

Fig.8. Flowchart of proposed Ranged-Based Routing (RBR) Protocol

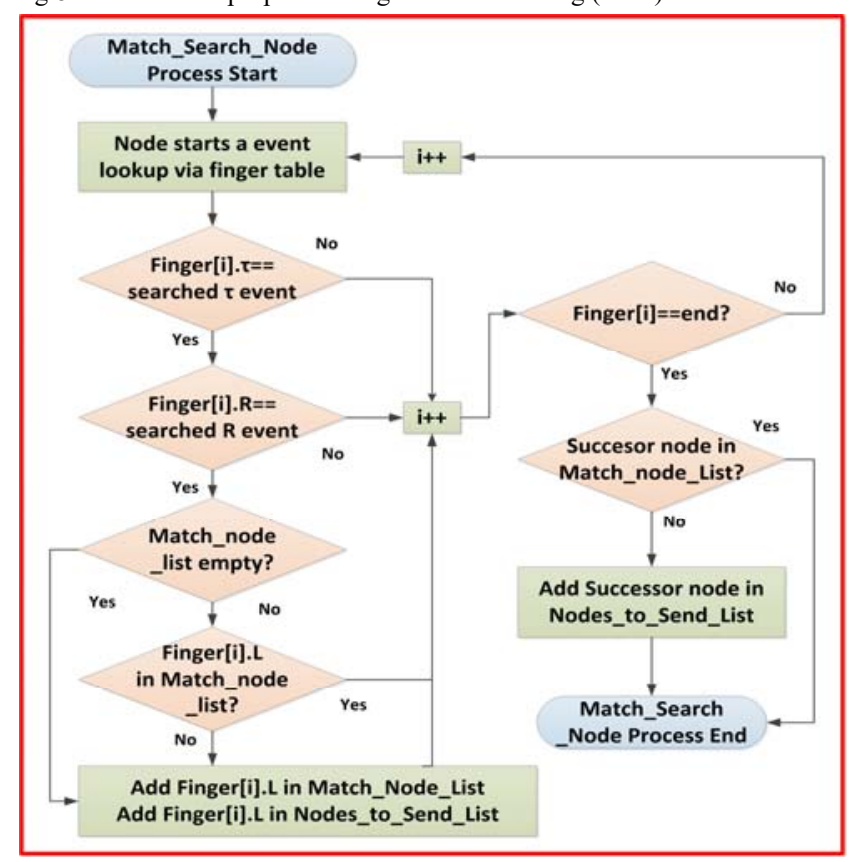

Fig.9. Detail of Match_Search_Node Process 


\section{Application Layer}

Since microgrids are inherent distributed systems, the event notification middleware have been designed following a distributed architecture. To achieve that, each peer in the system acts as a Notification Broker (NB), in other words, each peer implements a notification interface and the whole system acts as a single NB. As a result, the interface will not be a bottleneck, and the system will not have a single point of failure. Moreover, a P2P design avoids the need for centralized control and gives the flexibility to join or leave the system at any time. When a new node joins the pub/sub network, it contacts with its predecessor and successor and send its event types and subscription ranges that are interested in. When an event in the microgrid occurs, a node in the network publishes this event to the matching nodes in the ring. Upon receiving a publication, the node looks for the matched subscribers and forwards the publication according to the RBR protocol. It also sends the node list that has been received to avoid redundant search messages. An event matches a subscription if and only if the event type is matched and the Hilbert index of the cell for the publication is included in one of the intervals representing the subscription. For unsubscribing, the procedure is exactly the opposite of the subscribing process. The leaving node notify the leaving event to its predecessor and successor and the remaining nodes update its finger table. Besides, the protocol has selforganization capabilities. For this, Chord runs a stabilize algorithm in each node every 60 milliseconds to keep the list of predecessors and successors and to confirm that the ring integrity has not been corrupted. The subscriptions are actualized every 60 milliseconds in order to add or remove subscriptions. In this case, if a new subscription is merged by an existing subscription without changes in augmented subscription, it is not forwarded. Moreover, the proposed event notification middleware has a certain security degree in terms of integrity of data. This feature is achieved by implemented redundant links and self-reconfiguration methods. However, the system could be damaged by cyberattacks such as Sybil or Eclipse [40], [41]. Thus, additional security procedures such as tapping should be studied and implemented to reduce the sensitivity to malicious attacks. These aspects are out of the scope of this paper, which is focused on improving the efficiency of the microgrids middleware.

To illustrate the interest of the proposed middleware in microgrid environments, let us see an example of application. Let us assume that the event type "island mode" in microgrids is defined by two attributes: AC voltage and frequency. This is because in island mode, the grid voltage and frequency must be controlled to keep these variables in a desirable area. In accordance with European Standard EN50160 [42], the normal operating conditions for systems with no synchronous connection to an interconnected system (island mode) are described in table IV.
TABLE IV

VOLTAGE AND FREQUENCY VALUES FOR ISLAND MODE STABLISHED BY

\begin{tabular}{|l|l|l|}
\multicolumn{3}{|c|}{ EN50160 STANDARD } \\
\hline Range\#1 & Voltage & Frequency \\
& $\begin{array}{l}\text { 230 Volts } \pm 10 \% \\
\text { (i.e. } 207<\mathrm{V}(\text { volts })<253) \\
\text { during } 95 \% \text { of a week }\end{array}$ & $\begin{array}{l}50 \mathrm{~Hz} \pm 2 \% \\
\text { (i.e. } 49<\mathrm{f}(\mathrm{Hz})<51) \\
\text { during } 95 \% \text { of a week }\end{array}$ \\
\hline Range\#2 & $\begin{array}{l}\text { 230Volts }+10 \% /-15 \% \\
\text { (i.e.195.5<V(volts) }<253) \\
\text { during } 100 \% \text { of the time }\end{array}$ & $\begin{array}{l}50 \mathrm{~Hz} \pm 15 \% \\
(\text { i.e. } 42.5<\mathrm{f}(\mathrm{Hz})<57.5) \\
\text { during } 100 \% \text { of the } \\
\text { time }\end{array}$ \\
\hline
\end{tabular}

Figure 10 shows the operation zones that are defined as normal by the standard. Note that they are three zones clearly delimited. In the first one (zone \#1) the microgrid is always operating in normal conditions. The zone \#3 corresponds to abnormal conditions. Finally, in zone \#2 the operating conditions are classified as normal or abnormal depending on the conditions that table $\mathrm{V}$ specifies, so the time that the system is into zone\#2 shall be monitored. Therefore, it is interesting to define as an event and create a subscription when the system is operating in zone \#2.

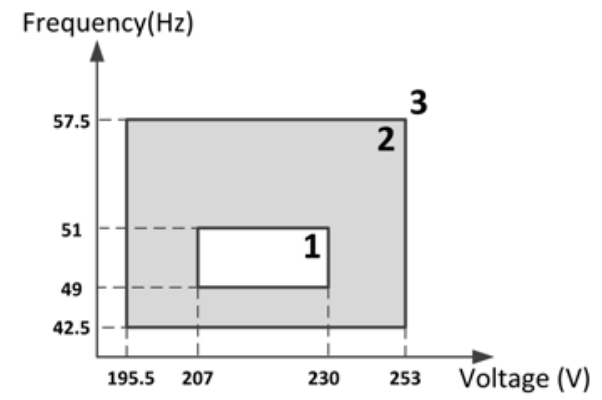

Fig.10. Operation zones in island mode defined by EN50160 standard

Thus the system for this example could be made as follows. On one hand, table $\mathrm{V}$ defines the four original subscriptions, $s_{1}, s_{2}, s_{3}$ and $s_{4}$, with its event type and its corresponding predicates. Note that the predicates associated to these subscriptions correspond to the operating conditions of interest (zone \#2). The subscription to the other zones could be carried by following a similar procedure.

TABLE V

SUBSCRIPTION EXAMPLE

\begin{tabular}{|c|c|}
\hline \multicolumn{2}{|c|}{$\begin{array}{c}\text { Subscription and predicates } \\
\tau=\text { Island Mode }\end{array}$} \\
\hline $\begin{array}{c}\text { Subscription } s_{1}: \\
\tau(195.5 \leq \text { voltage } \leq 207 \cup \\
42.5 \leq \text { current } \leq 57.5)\end{array}$ & $\begin{array}{c}\text { Subscription } s_{2}: \\
\tau(195.5 \leq \text { voltage } \leq 253 \cup \\
42.5 \leq \text { current } \leq 49)\end{array}$ \\
\hline $\begin{array}{c}\text { Subscription } s_{3}: \\
\tau(230 \leq \text { voltage } \leq 253 \cup \\
42.5 \leq \text { current } \leq 57.5)\end{array}$ & $\begin{array}{c}\text { Subscription } s_{4}: \\
\tau(195.5 \leq \text { voltage } \leq 253 U \\
51 \leq \text { current } \leq 57.5)\end{array}$ \\
\hline
\end{tabular}

Figure 11 illustrates the proposed example in addition with three possible publications $\mathrm{p}_{1}, \mathrm{p}_{2}, \mathrm{p}_{3}$ each one corresponding to the described operation zones. The resulting augmented subscription $S_{\text {aug }}$ is the rectangle that has intersection with the original subscriptions.

Therefore, the set off cells that form the augmented subscription, $S_{-}$aug, is

$\{0,1,14,15,16,19,3,2,13,12,17,18,4,7,8,11,30,29,5,6,9,10,31,2$ $8,58,57,54,53,32,35,59,56,55,52,33,34,60,61,50,51,46,45\}$. It is worth to point out that $\mathrm{p} 3$ will be detected as a false 
positive, but the system knows that it corresponds to the zone\#1 so it can be easily eliminated from the list of interest.

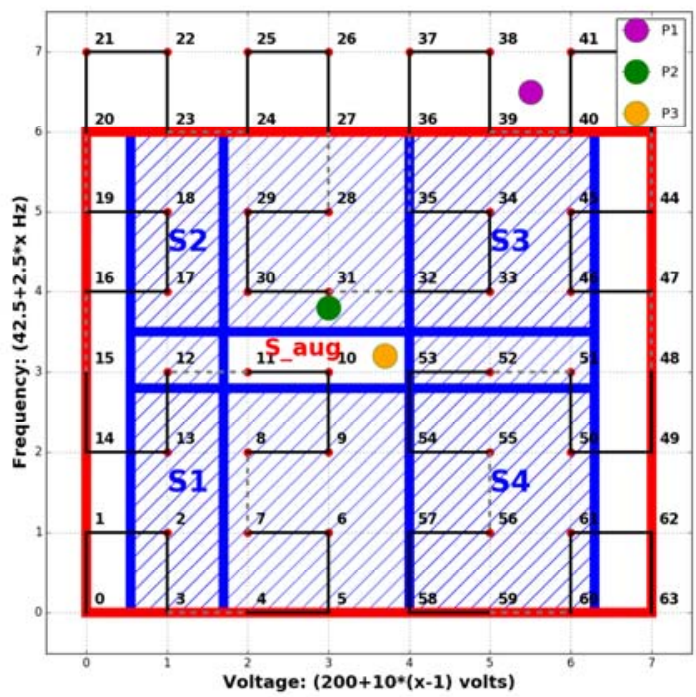

Fig.11. Sample publication and subscription indexing in a two dimensional space, $\mathrm{H}_{3}^{2}$

\section{EXPERIMENTAL RESULTS}

The proposed routing engine and matching system using Hilbert space have been evaluated and compared with the routing and matching methods based on multicast, which are used traditionally in microgrids. To carry out this evaluation, an experimental setup (shown in Figure 12) has been built up to measure the main performance indicators of the network that where defined in section II: subscriptions flexibility, latency, bandwidth consumption, routing performance, efficiency and computational resources saving. Conceptually, there is not a limitation on the number of nodes that can be interconnected by means of the proposed distributed techniques. However, some physical limitations will appear in a practical implementation, which are mainly associated to the limits of the communication channel (bandwidth, baud rate, noise, etc...). The experimental setup is able to manage up to 20 nodes.

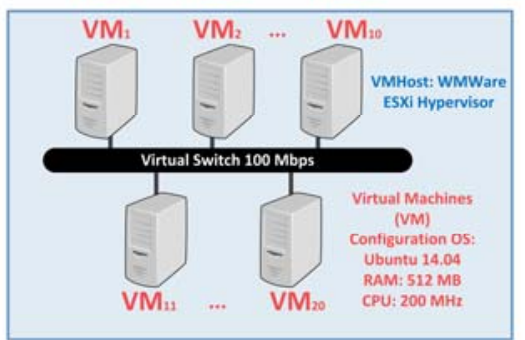

Fig.12. Schematic drawing of the experimental setup

This configuration agrees with the one of the embedded systems that we have initially chosen to migrate from virtual to physical machines, so the experimental results that are presented are realistic and valuable to preliminarily validate the proposed solution. All tests were run for 15 minutes with publishers publishing an event every 10 seconds. The Hilbert matrix used for experimental evaluation has been $\mathrm{H}_{3}^{2}$.

It is worth noting that the proposed middleware should be implemented on each one of the nodes. Therefore, each node is interconnected with its corresponding electric devices of the microgrid and also with the communication infrastructure. In this way, the proposed communication system is adaptable to any kind of microgrid configuration and network topologies.

\section{A. Subscriptions Flexibility}

As it has been explained before, there are two major classes of publisher/subscriber systems: topic-based and contentbased.

On the one hand, in topic-based systems, subscribers join a group containing a topic of interest. Publications are identified by specific topics. Therefore, all publications related to that topic are broadcasted to all nodes of the specific group.

On the other hand, in content-based systems, the subscribers can accurately specify their interest using a set of predicates. In other words, a subscription is a request formed by a set of constraints. Therefore, in the proposed system, the Hilbert filling curve is used to map a multi-dimensional space to a compact Hilbert key, which specifies the pair (attribute, value) that defines the constrains. Whit both the Hilbert dimension and order, the attributes and the values are specified, respectively. In this way, two pairs of (attribute, value) can be represented with a Hilbert key by using 2D-n order Hilbert curve. Similarly, three pairs of (attribute, value) by using 3D-n order, and so on (see Figure 13).
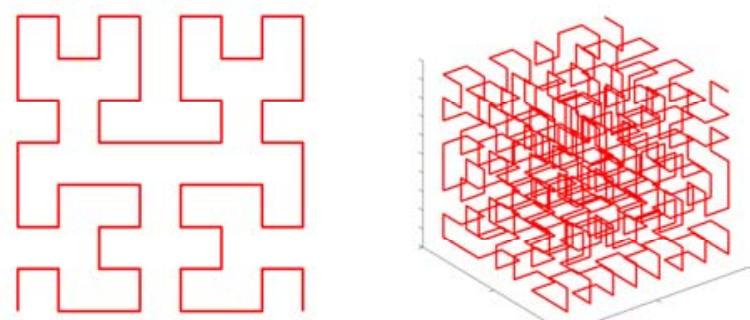

Fig.13. Hilbert space-filling curve (2D-3rd order and 3D-3rd order from left to right)

By using Hilbert space filling curve a subscription with $\mathrm{N}$ constraints can be encode as a unique Hilbert key. Once the Hilbert key is package, the RPL protocol will be able to send the message only to those nodes whose subscription matches with all the publication constraints. In other words, all nodes that are subscribed to that Hilbert key.

To compare the performance of both techniques, the topicbased approach has been emulate as content-based one as follows:

i) Firstly, the publication with the specified topic is broadcasted to all nodes that are subscribed to the specific topic group.

ii) Once the nodes are subscribed, they inform to the publishers about their subscription. Therefore, the first constraint will be sent only to those nodes that have been subscribed to that topic.

iii) The nodes that match with the topic and with the first constraint also inform to the publishers. In this way, the 
publishers send the second constraint only to those nodes that match these conditions. This process continues by sending publications for the $\mathrm{N}$ constraints.

Figure 14 shows a comparative study of the number of the interchanged messages that are needed to send a set of publications. The results obtained with Hilbert content-based are compared to the ones achieved by topic-based in three cases: no constraints, with one constraint and with two constraints.

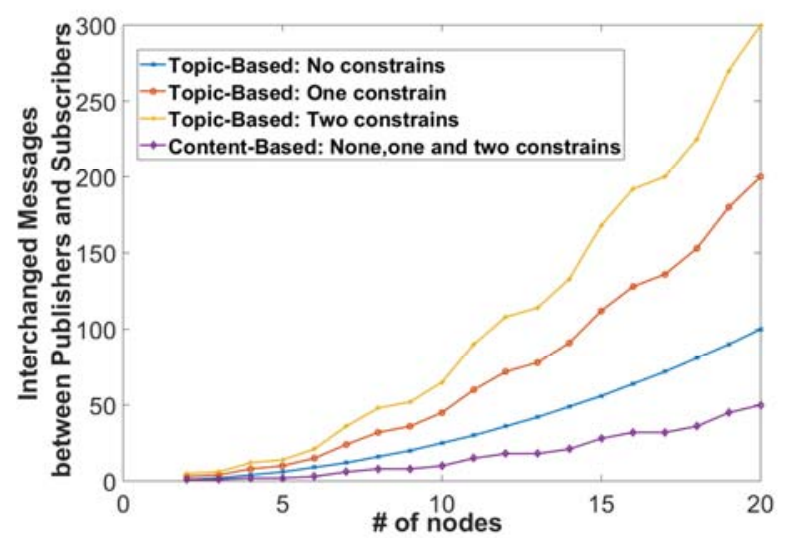

Fig.14. Costs of publishing messages in relation with the publications constraints by using topic and content based approaches.

As it can be seen in Figure 14, the number of interchanged messages by using the filtered topic-based approach depends on both, the number of nodes in the network and on the number of constraints in the publication. Indeed, for sending $\mathrm{N}$ constraints the publishers need to know the nodes that are subscribed to the first $\mathrm{N}-1$ constraints to verify that the subscription match to all the publication constraints. This fact generates more network traffic. On the contrary, with the filtered Hilbert content-based approach, $\mathrm{N}$ constraints can be defined in a unique Hilbert key. Therefore, the number of interchanged messages only depends on the number of nodes in the system and it does not depend on the number of publication constraints. The higher expressiveness and flexibility of content-based approach reduce the need for network resources. The network robustness and scalability improve due to the notifications are only sent to the actual subscribers.

\section{B. Network Performance}

This section focuses on the two most critical components that dictate the performance of network in microgrids: delays and bandwidth. Regarding network delay, Figure 15 shows the overall End-to-End (ETE) delay, that is the time elapsed from the publication source to the last subscriber, in average value for pub/sub solutions based on multicast vs. Hilbert. This figure shows that ETE delay for the proposed system is lower than the one that offers the pub/sub system based on multicast. Note that the proposed system reduces the subscription-forwarding load at each node. Therefore, the ETE delay is lower than the achieved one by using traditionally pub/sub based on multicast methods. As it is shown, the proposed pub/sub middleware improves the ETE delay between $35 \%$ and $48 \%$, and the improvement increases as more nodes connect to the network. The total ETE delay for 20 nodes achieved by means of the proposed pub/sub middleware is around $270 \mathrm{~ms}$, while with pub/sub based on multicast it is around $530 \mathrm{~ms}$. Thus, the average ETE delay per node in the proposed system can be estimated around 14 ms. This means that the average latency to process a publication in a pub/sub based on Hilbert middleware is around $14 \mathrm{~ms}$, which meet the network microgrid requirements that were outlined in Table I.

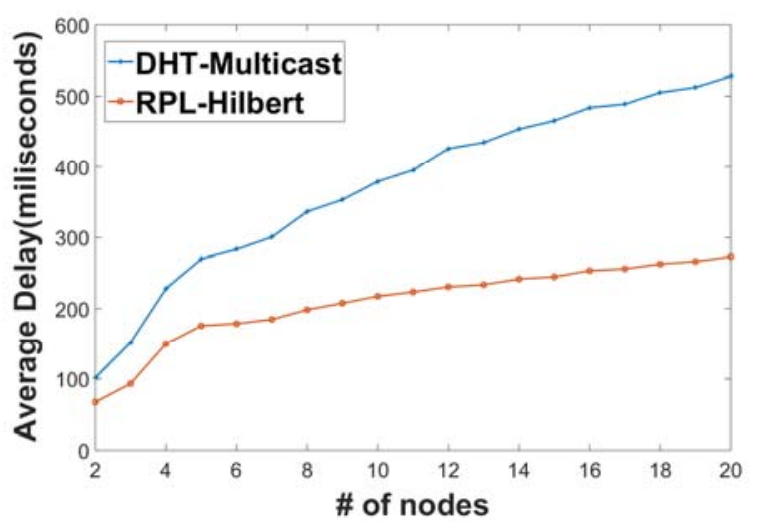

Fig.15. Comparison of average ETE delay vs increasing number of nodes for pub/sub based on multicast and Hilbert

Related to bandwidth measurements, Figure 16 shows the average bandwidth consumption in the network for different scales. For that three different scales are considered with testbeds of 5, 10 and 20 nodes connected to the network, and the bandwidth consumption of the proposed pub/sub system against that of pub/sub based on multicast routing method has been compared.

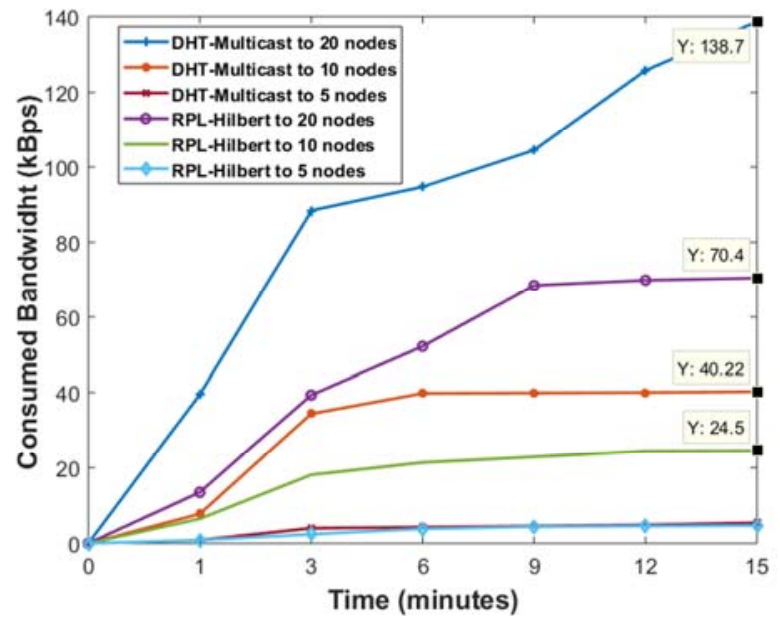

Fig.16. Average total bandwidth consumption in time for different scale

As it can be seen in Figure 16, the bandwidth consumption with the proposed pub/sub method increases less rapidly that with pub/sub methods based on multicast. Overall, the average total bandwidth consumption over 15 minutes decreases by up to $39.08 \%$ for 10 nodes and $49.2 \%$ for 20 nodes where using the proposed pub/sub middleware. These results could justify the use of the proposed pub/sub system based on Hilbert at the application layer as better use of available bandwidth is produced due to the traffic is reduced 
by eliminating redundant transmission. This redundant transmission has been reduced by applying subscription merged which can greatly reduce the subscription dissemination traffic. In addition, the bandwidth savings increase with the size of the network, ensuring more scalable communications.

\section{Routing Performance}

The main benefits of the proposed RPL protocol are a reduction in both the network traffic (Figure 17) and the routing time (Figure 18). The traffic in the network has been measured by averaging the total bandwidth consumption in kilobytes per second. Figure 17 shows the average total network traffic (in consumed bandwidth) for 20 nodes at 15 minutes.

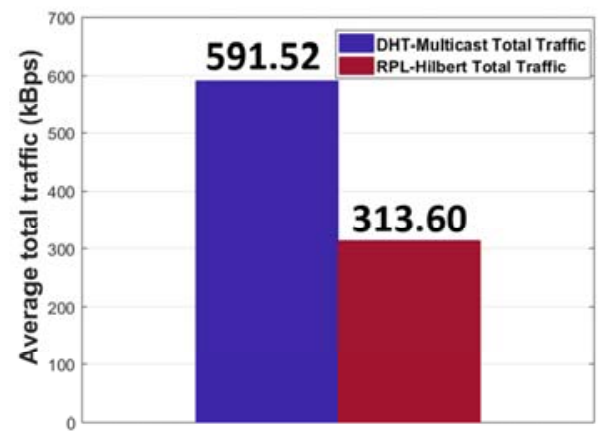

Fig.17. Consumed bandwidth on 20 nodes network for pub/sub system based on multicast and Hilbert routing.

As Figure 17 shows, the RPL-Hilbert technique consumes near two times less traffic that multicasting. The reason is that, by using RPL protocol, the neighbor routing tables of each node have information about their subscriptions. In this way, each publication is delivered only to the subscribed nodes and not to the subscribed clusters as multicast routing does. Consequently, a network traffic reduction is achieved. In addition, RPL protocol has self-organization capability due to the overlay Chord infrastructure. With this feature, each node knows the disposition of their neighbors, which produces an effective method for publication and subscription propagation and allows a reduction in the routing time. Figure 18 shows the average matching time of publications against subscriptions. The average matching time is the average time to route the publications to the matched subscriber. They have been generated 3000 subscriptions (50 random subscriptions for each subscriber node) to evaluate the publication matching time.

As it can be seen in Figure 18, it takes around 13 milliseconds to route a publication against 3000 subscriptions by using the proposed RPL-Hilbert algorithm. With DHT Multicast this time is around 29 milliseconds. The matching time does not significantly increase as the number of subscriptions grows. This indicates that the proposed approach is suitable for large scale publish/subscribe systems and can efficiently process a large number of messages (publications and subscriptions).

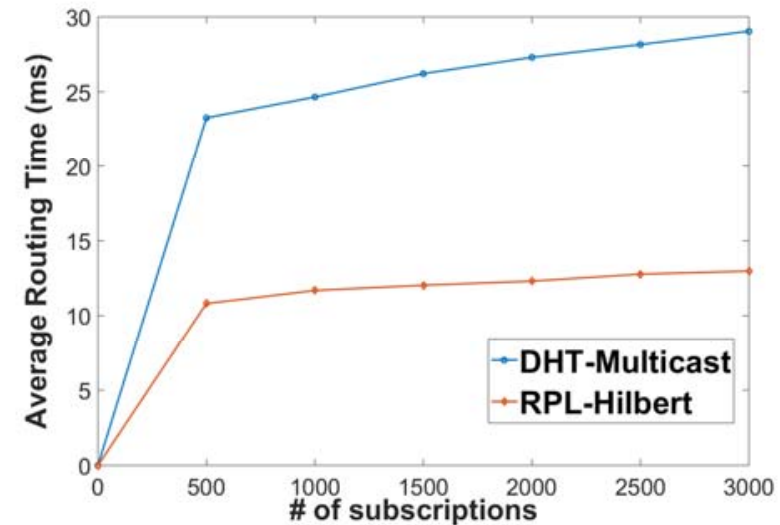

Fig.18. Average Publication Matching Time

\section{Efficiency: False Positives}

Figure 19 is the result of one of the most important performance metric for efficiency, false positives. A false positive is defined as a message received by the node that is not interested in the message. This figure shows the total average number of received messages per node. This refers to the total publication messages that receive the nodes. Moreover, the figure also shows the percentage of false positive for this quantity of received messages. As it can be seen, both average received message and false positives with the proposed method results in considerable reduction regarding pub/sub based on multicast routing approaches. The percentage of false positive decreases as network grows, which ensure more efficient communications. This is due to the use of Hilbert mapping for routing and merging matching that allows the proposed pub/sub system having more chance to forward an event to a better neighbor. Note that the event is embedded in the routing table and the event matching has more probability of success. Moreover, by using the minimum-bounding rectangle as merging technique, the area for false positives is reduced and the number of the unwanted publications is small.

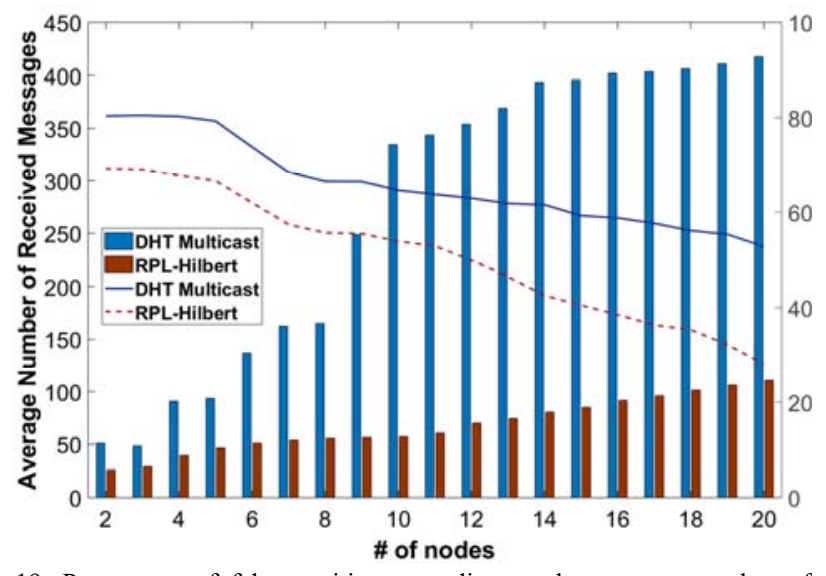

Fig.19. Percentage of false positive according to the average number of received publications per node

\section{E. Computational Resources Savings}

System memory and CPU are critical resources for the operation and performance of any software system. The use of 
the merging technique allows reducing the routing subscription tables' size. Thus the processing and storage memory requirements that are used by the nodes are also reduced. Figures 20 and 21 show the average amount of active memory and CPU load, respectively, on a network of 20 peers over 15 minutes, with both the proposed pub/sub system and the one based on multicast.

Multicast needs more memory as the scale increases, up to $130 \mathrm{MB}$ of memory for 20 nodes over 15 minutes, while pub/sub based on Hilbert requires $85 \mathrm{MB}$ in the same conditions. Similar to the memory analysis, the CPU savings increases with the size of the network. Multicast solutions use around $74 \mathrm{MHz}$ of CPU for 20 nodes over 15 minutes while Hilbert solution uses around $56 \mathrm{MHz}$.

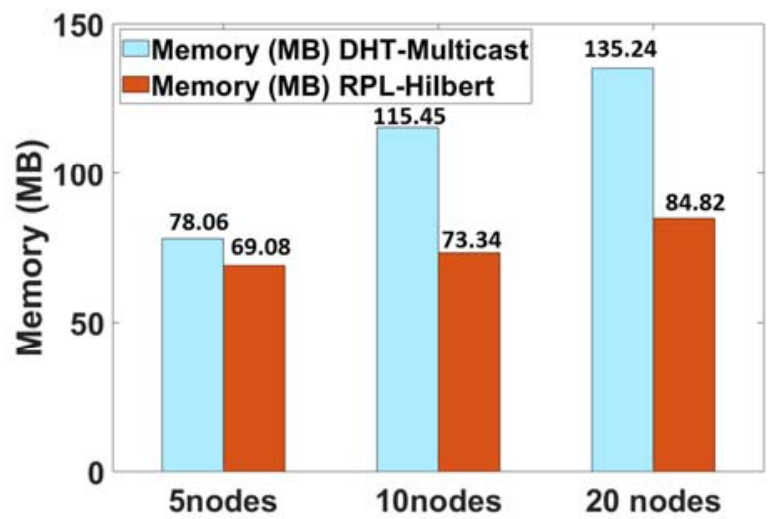

Fig.20. Average Active Memory used (in MB). Measurements have been taken for three different scales for pub/sub solutions based on multicast and Hilbert over 15 minutes.

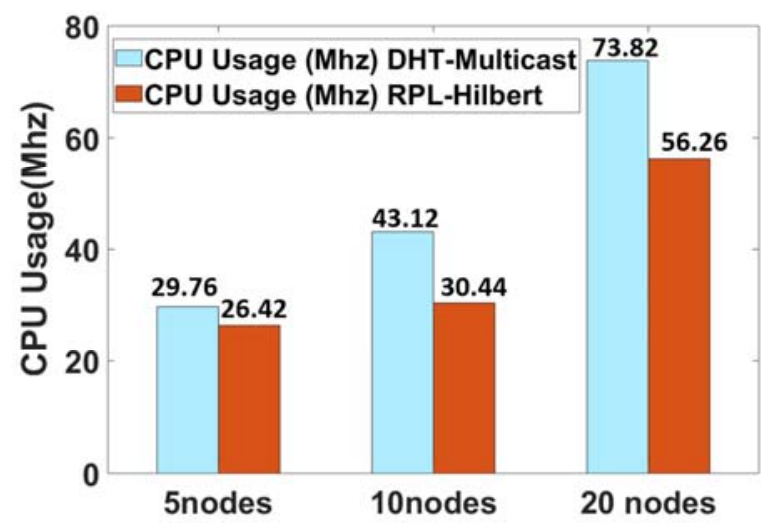

Fig.21. Average CPU usage (in MHz). Measurements have been taken for three different scales for pub/sub solutions based on multicast and Hilbert over 15 minutes.

The showed results confirm that the proposed system can be implemented with a reasonable computational resources usage which allows its implementation in embedded systems

\section{CONCLUSION}

In this paper a new event notification middleware for content-based pub/sub over peer-to-peer network suitable for large-scale microgrids has been presented. The proposed middleware uses multidimensional indexing to represent publications and subscriptions in one-dimensional space through the Hilbert space-filing curve. Based on this representation, routing, merging and matching algorithms have been developed. On one hand, the ranged based routing protocol (RBR) developed can construct and efficient event dissemination while it has desirable properties such as selforganization and scalability. On the other hand, the proposed merging and content matching algorithms improve the traffic performance and reduce the false positives rate due to the minimization of the space of participation nodes that have no interest in the event, providing a higher level of reliability guarantee. Experimental results have shown that middleware based on the Hilbert space improves efficiency, reduces the overall network traffic and latencies and achieves better computer resources savings. Those improvements are needed to meet the demanding communications requirements for microgrids. The tests have been carried out by means of virtual machines by taking into account most of the system physical limitations. Therefore, it can be concluded that the proposed solutions are an interesting approach to the problem of communications in smart microgrids.

\section{REFERENCES}

[1] M. S. Golsorkhi, Q. Shafiee, D. Lu and J. M. Guerrero, "A distributed control framework for integrated photovoltaic-battery based islanded microgrids," IEEE Trans. Smart Grid, vol.8, no.6, pp.2837-2848, 2017.

[2] L. Ren, Y. Qin, B. Wang, P. Zhang, et al., "Enabling resilient microgrid through programmable network," IEEE Trans. Smart Grid, vol.8, no.6, pp.2826-2836, 2017.

[3] H. Pourbabak, T. Chen, B. Zheng, and W. Su, "Control and Energy Management Systems in Microgrids," Clean Energy Microgrids (S. Obara, ed.), The Institution of Engineering and Technology (IET), 2017.

[4] H. Pourbabak, J. Luo, T. Chen and W. Su, "A novel consensus-based distributed algorithm for economic dispatch based on local estimation of power mismatch," IEEE Trans. Smart Grid, 2017.

[5] J. F. Martínez, J. Rodríguez-Molina, P. Castillejo and R. De Diego, "Middleware architectures for the smart grid: survey and challenges in the foreseeable future," Energies, vol. 6, no. 7, pp. 3593-3621, 2013.

[6] M. Macarulla, M. Albano, L. L. Ferreira and C. Teixeira, " Lessons learned in building a middleware for smart grids," Journal of Green Engineering, vol. 6, no. 1, pp. 1-26, 2016.

[7] A. Werth, A. Andre, D. Kawamoto, T. Morita, et al., "Peer-to-peer Control System for DC Microgrids," IEEE Trans. Smart Grid, 2016.

[8] T. Morstyn, A. Teytelboym, and M. McCulloch, "Bilateral contract networks for peer-to-peer energy trading," IEEE Trans. Smart Grid, 2018.

[9] B. Duan, C. Tan and Y. Liu, "Towards Harmonization IEC61850 and Intelligent Agents: Characteristic Analysis of Service Tracking," Energy and Power Engineering, vol. 5, no.04, pp. 1308-1311, 2013.

[10] S. Gupta, I. Ali, M. S. Thomas and S. S. Hussain, "Recent advancements in substation automation systems based on information and communication technologies," Communication and Computing Systems, 2017.

[11] F. Cao and J. P. Singh, " Efficient event routing in content-based publish-subscribe service networks," PrOc. IEEE INFOCOM'04, vol. 2, pp. 929-940, 2004.

[12] A. Bari, J. Jiang, W. Saad and A. Jaekel, " Challenges in the smart grid applications: an overview," Int. J. Distrib. Sensor Netw., vol.10, no.2, pp. 1-14, 2014.

[13] G. Banavar, T. Chandra, B. Mukherjee, J. Nagarajarao, R. E. Strom and D. C. Sturman, "An efficient multicast protocol for content-based publish-subscribe systems," Proc. ICDCS 99Intl Conf. Distributed Computing Systems, 1999.

[14] H. Jafarpour, S. Mehrotra, N. Venkatasubramanian and M. Montanari, "Mics: an efficient content space representation model for publish/subscribe systems," Proc. of DEBS'09, ACM, pp.1-12, 2009.

[15] G. T. Edwards, D. C. Schmidt and A. Gokhale, "Integrating publisher/subscriber services in component middleware for distributed 
real-time and embedded systems," Proc. of the 42nd annual Southeast regional conference. ACM, pp. 171-176, 2004.

[16] W. Meng, X. Wang, and S. Liu, "Distributed load sharing of an inverterbased microgrid with reduced communication," IEEE Trans. Smart Grid, 2016.

[17] J. Zhou, H. Zhang, Q. Sun, D. Ma and B. Huang, "Event-Based Distributed Active Power Sharing Control for Interconnected AC and DC Microgrids," IEEE Trans. Smart Grid, 2017.

[18] D. Tam, R. Azimi and H. A. Jacobsen, "Building content-based publish/subscribe systems with distributed hash tables," Proc. Int'l Wksp. Databases Info. Sys. and Peer-to-Peer Computing, vol. 2944, pp. 138-152, 2003.

[19] Li, G., Muthusamy, V., and Jacobsen, H. A. "Adaptive content-based routing in general overlay topologies," In Proceedings of the 9th ACM/IFIP/USENIX International Conference on Middleware (pp. 121). Springer-Verlag New York, Inc..2008

[20] S. Sučić, J. G. Havelka and T. Dragičević, "A device-level serviceoriented middleware platform for self-manageable DC microgrid applications utilizing semantic-enabled distributed energy resources," Int. J. Electr. Power Energy Sys., vol. 54, pp. 576-588, 2014.

[21] A. Bose, "Smart transmission grid applications and their supporting infrastructure," IEEE Trans. Smart Grid, vol.1, no.1, pp.11-19, 2010.

22] M. Albano, L. Ferreira, L. Pinho, A. Alkhawaja, "Message-oriented middleware for smart grids," Computer Standards \& Interfaces, Elsevier,vol.38, pp. 133-143, 2015.

[23] L. Zhou, and J. J. Rodrigues, "Service-oriented middleware for smart grid: Principle, infrastructure, and application," IEEE Commun. Mag., vol.5, no.1, pp.84-89, 2013.

[24] H. Ali, A. A. Mamun, and S. Anwar, "A Comprehensive Study of Advancement of Electrical Power Grid and Middleware Based Smart Grid Communication Platform," International Journal of Advancements in Technology, 2016.

[25] M. Starke, A. Herron, D. King and Y. Xue, "Implementation of a Publish-Subscribe Protocol in Microgrid Islanding and Resynchronization with Self-Discovery," IEEE Trans. Smart Grid, 2017.

[26] T. A. Youssef, A. T. Elsayed and O. A. Mohammed, "Data Distribution Service-Based Interoperability Framework for Smart Grid Testbed Infrastructure," Energies, vol. 9, no. 3, pp. 150, 2016.

[27] S. Bhowmik, "Content-based routing in software-defined networks," Ph.D. dissertation, Dept. Elect. And Inform. Eng., Stuttgart, Univ.,Germany, 2017.

[28] A. Vulgarakis and C. Seceleanu, "Embedded systems resources: Views on modeling and analysis," Proc. Annu. Int. Comput. Softw. Appl. Conf., vol.0, pp. 1321-1328, 2008.

[29] P. Kansal and A. Bose, "Bandwidth and latency requirements for smart transmission grid applications,” IEEE Trans. Smart Grid, vol.3, no.3, pp. 1344-1352, 2012.

[30] IEC Communication Networks and System in Power Utility Automation, IEC Standard. 61850-7-420, 2009.

[31] IEEE Communication Delivery Time Performance Requirements for Electric Power Substation Automation; IEEE Standard 1646, 2005.

[32] J. Rodríguez-Molina, J. F. Martínez, P. Castillejo and G. Rubio, "Development of Middleware Applied to Microgrids by Means of an Open Source Enterprise Service Bus," Energies, vol.10, no.2, pp.172, 2017.

[33] K. H. Gjermundrod, D. E. Bakken, C. H. Hauser and A. Bose, "GridStat: A flexible QoS-managed data dissemination framework for the power grid," IEEE Trans. Pow. Del., vol.24, no.1, pp. 136-143, 2009.

[34] S. Marzal, R. Salas, R. González-Medina, G. Garcerá and E. Figueres, "Current challenges and future trends in the field of communication architectures for microgrids," Renew. Sustain. Energy Rev., vol.81, pp.3610-3622, 2017.

[35] R. Baldoni and A. Virgillito, "Distributed event routing in publish/subscribe communication systems: a survey," DIS, Universita di Roma La Sapienza, Tech. Rep, 2005.

[36] L. Opyrchal, M. Astley, J. Auerbach, G. Banavar, et al., "Exploiting IP multicast in content-based publish-subscribe systems," Proc. Of Middleware, pp. 185-207, 2000.

[37] B. Hayes, "Crinkly curves," AMERICAN SCIENTIST, vol. 101, no. 3, pp. 178-183, 2013.
[38] B. Moon, H. V. Jagadish, C. Faloutsos and J. H. Saltz, "Analysis of the clustering properties of the Hilbert space-filling curve," IEEE Trans. Knowledge and Data Eng., vol.13, no.1, pp.124-141, 2001

[39] N. J. Navimipour and F.S. Milani, "A comprehensive study of the resource discovery techniques in Peer-to-Peer networks," Peer-to-Peer Netw. Appl., vol. 8, no. 3, pp. 474-492, 2015.

[40] L. Wang, "Attacks Against Peer-to-peer Networks and Countermeasures", TKK Seminar on Network Security, no. 12, pp. 5161, 2006.

[41] G. Jaideep and B. P. Battula, "Survey on the present state-of-the-art of P2P networks, their security issues and counter measures," Int. J. Appl. Eng. Res, vol.11, no.1, pp. 616-620, 2016.

[42] CENELEC Voltage characteristics of electricity supplied by public distribution systems, CENELEC EN50160, 2007. 